\title{
Formazione delle parole e terminologia grammaticale: proposte per il mondo della scuola
}

\section{Word formation and grammatical terminology: proposals for schools}

\author{
Maria G. Lo Duca \\ Università di Padova \\ $\square$ mariag.loduca@unipd.it
}

Riassunto / II saggio si pone l'obiettivo di affrontare e sperabilmente chiarire alcuni snodi problematici relativamente al settore della formazione delle parole in italiano. Si presenta come una breve rassegna di parole tecniche - composizione, parole composte e parole polirematiche, conversione, parasintesi - che vengono illustrate attraverso degli esempi pertinenti, allo scopo di chiarire i fatti linguistici cui esse rimandano. Interlocutore ideale di questo saggio è il docente di italiano, o lo studente universitario che si prepari a diventarlo. A questo lettore ideale si offrono dati e ragionamenti che tentano di spiegare almeno alcune delle principali acquisizioni della ricerca moderna su questi temi, nella speranza che il chiarimento concettuale si accompagni ad una più sicura professionalità docente, in grado di adottare a ragion veduta l'inevitabile terminologia e scegliere con cognizione di causa i testi scolastici.

Parole chiave: conversione; composizione; formazione delle parole; parole polirematiche; parasintesi.

Abstract / This paper aims to address and hopefully clarify some problematic issues related to Italian word formation. The contribution is presented as a brief review of technicalities - such as composition, compound words and multiword expressions, conversion, parasynthesis - which are illustrated through pertinent examples to clarify the linguistic facts they refer to. Italian language teachers, either already in charge or still graduating, are the main addressees of this essay. These ideal readers are offered data and

\footnotetext{
(C) 2021 Maria G. Lo Duca. Questo è un articolo Open Access, sottoposto a un processo di revisione tra pari a doppio cieco, pubblicato dal Centro competenze didattica dell'italiano lingua di scolarizzazione e dal Servizio risorse didattiche e scientifiche, eventi e comunicazione del DFA-SUPSI in collaborazione con l'Alta scuola pedagogica dei Grigioni. L'articolo è distribuito sotto i termini della Licenza Creative Commons Attribuzione - Non commerciale - Non opere derivate 4.0 Internazionale (CC BY-NC-ND 4.0).
} 
arguments that attempt to explain at least some of the main achievements of modern research on these issues, hoping that with these tools the conceptual clarification will be accompanied by a more confident teaching professionalism - namely by adopting the suitable terminology and making an informed choice of school texts.

Keywords: conversion; composition; word formation; multiword expressions; parasynthesis.

\section{Premessa}

Mi sono di recente occupata di formazione delle parole, un settore su cui sono a più riprese tornata nella mia vita di studiosa: a cominciare dalla mia tesi di dottorato, poi diventata libro (Lo Duca, 1990), in cui in verità mi occupavo di acquisizione di alcuni procedimenti derivativi dell'italiano, per finire a quello che, nelle mie intenzioni, vorrebbe essere un resoconto agile e aggiornato del campo di studi, offerto non tanto agli studiosi, quanto agli studenti di lettere e di linguistica, e agli insegnanti di italiano (Lo Duca, 2020). Già in quest’ultima opera mi sono dovuta porre il problema della terminologia (e delle connesse partizioni), che ho risolto sulla base della letteratura di riferimento, senza pormi il problema degli usi scolastici, che ad uno sguardo superficiale sembrano peraltro abbastanza in linea con le scelte terminologiche degli studiosi del settore. Oggi ritorno su alcune questioni soprattutto terminologiche, per cercare di contribuire a chiarire e a mettere ordine. Sono infatti convinta che la terminologia non è una variabile di poco conto nello studio di una disciplina, e condivido in pieno un'idea di Adriano Colombo, espressa con la solita lucidità, cioè che «dietro l'approssimazione terminologica ci può essere una certa approssimazione concettuale» (Colombo, 2009, p. 285). Nell'intento, quindi, di chiarire i concetti, scriverò del modo di denominarli.

Ho organizzato questo intervento intorno ad alcune parole tecniche di questo settore della grammatica dell'italiano. All'interno poi dei vari paragrafi mi soffermerò su alcuni casi problematici che sono diversamente etichettati dai diversi autori, nella speranza di contribuire a fugare almeno in parte qualche dubbio o insicurezza. 


\section{Formazione delle parole o morfologia lessicale}

Cominciamo dai termini che più spesso si usano per designare il campo disciplinare di cui ci occuperemo. Le due locuzioni designano in realtà lo stesso ambito della grammatica. Forse "formazione delle parole" è più trasparente, ma indica né più né meno che l'oggetto di studio della morfologia lessicale. La quale è uno dei due grandi rami in cui viene solitamente suddivisa la morfologia: lessicale e flessiva.

Della morfologia flessiva non parleremo, ma è quella di gran lunga più nota e praticata nella scuola: si occupa, come è risaputo a) della forma delle parole, normalmente suddivise in sottoclassi, le tradizionali "parti del discorso", oggi più spesso denominate "categorie lessicali” o "classi di parole”; b) dei cambiamenti di forma che le parole (variabili) subiscono, secondo i parametri del genere e del numero per i nomi, gli articoli, gli aggettivi e i pronomi; della persona, del tempo, del modo, della diatesi e dell'aspetto, per i verbi. Questo ramo della morfologia ha goduto e gode di grande attenzione e considerazione in ambito scolastico, tanto da essere denominato "morfologia" tout court.

La morfologia lessicale copre invece l'ampio settore della formazione delle parole, e studia i procedimenti attraverso cui, in una data lingua, si formano le nuove parole, a partire da materiale lessicale - parole, affissi - già presenti nella lingua. Ignora per lo più i fenomeni del prestito, a meno che le singole unità lessicali, prestate all'italiano da altre lingue, non risultino a loro volta coinvolte in procedimenti formativi di vario genere (jeans-eria, computer-ese, chatt-are, fine settimana, calco di week-end).

Nella scuola, nell'affrontare questo capitolo della grammatica dell'italiano, ci si ferma di solito alla derivazione e alla composizione, mentre ricevono poca o nessuna considerazione altri temi studiati dalla morfologia lessicale, altre possibilità dell'italiano, quali la conversione, la parasintesi, la retroformazione, la sostituzione, l'accorciamento, la reduplicazione, la formazione di sigle e di parole macedonia. Eppure sono fenomeni interessanti, alcuni marginali, altri molto produttivi e ossessivamente presenti nelle nostre vite ${ }^{1}$, e, a giudicare

1 Si pensi, solo per fare un esempio, all'imperversare recente di sigle, o acronimi, in tutti gli ambiti disciplinari, in primis nel linguaggio burocratico che accompagna in ogni suo atto la vita della scuola. 
dalle incertezze e dai veri e propri errori di etichettatura che vedo frequenti nei libri destinati alle scuole, non sarebbe male fare chiarezza almeno su alcuni di questi fenomeni e condurre su di essi, se è il caso, una riflessione mirata. E questo non per imparare qualche parola tecnica in più (obiettivo lontanissimo dai miei interessi), ma per individuare, attraverso le parole che li designano, oggetti linguistici degni di attenzione. Per adesso accade che i procedimenti che questi termini designano vengono o ignorati, o, il che è più grave, erroneamente etichettati dai libri di testo e ascritti di default all'area della derivazione o della composizione. Il che crea spesso delle contraddizioni evidenti con le sistemazioni che gli stessi libri di testo danno dei vari fenomeni, e, negli insegnanti e studenti più accorti, domande destinate a restare senza risposta.

Naturalmente anche per questo settore della grammatica bisognerebbe pensare ad una progressione dei contenuti e relative terminologie, con un graduale approfondimento dei temi e conseguente ampliamento del bagaglio terminologico che vadano di pari passo con la crescita cognitiva e linguistica degli studenti. Ma non prenderò in considerazione questo aspetto del problema, e dunque non mi chiederò se e quando un certo procedimento, e relativo termine che lo designa, vadano inseriti in un curriculum scolastico. Il mio intento di oggi è contribuire a svecchiare la cultura morfologica degli insegnanti, suscitando dubbi ma anche suggerendo qualche soluzione, e nel contempo alimentando, sperabilmente, il desiderio di saperne di più.

\section{Composizione, e dintorni}

Il capitolo della composizione trova sempre un suo spazio nei libri per le scuole. Normalmente vi sono elencate formazioni costituite da due parole aventi vita autonoma nel lessico, e il cui significato, unitario e ben riconoscibile, è composizionale, dato cioè dalla somma del significato delle due parole coinvolte: quindi, ad esempio, capoclasse (nome + nome, equivalente a 'capo della classe'), camposanto (nome + aggettivo, 'campo reso santo dalla presenza dei morti'), portaombrelli (verbo + nome, 'oggetto in cui si mettono gli ombrelli'), agrodolce (aggettivo + aggettivo, riferito a qualcosa 'che ha gusto insieme agro e dolce'), sempreverde (avverbio + aggettivo, riferito ad una pianta 'che è sempre verde, le cui foglie non cadono durante l'inverno'), capovolgere (nome + verbo, 
'voltare qualcosa in modo che ciò che prima era la testa si trovi nella parte inferiore'), e così via. Sono i casi più noti e assodati, che trovano di solito posto nelle grammatiche. Qualcuno vi aggiunge i composti con costituenti di origine greca o latina del tipo biologia, geografia, democrazia, che tanta diffusione e spazio hanno nelle discipline, e i cui elementi costitutivi (attenzione!) sono diversamente denominati dai diversi autori: "prefissoidi» e "suffissoidi» (Migliorini, 1963; Serianni, 2000; nella manualistica scolastica in genere); «semiparole» (Scalise in tutti i suoi lavori, e da ultimo in Scalise \& Bisetto, 2008); «confissi» (De Mauro, 1999-2000; Sgroi, 2003); infine, in modo più generico, «elementi formativi» (Iacobini, 2004, 2010; Lo Duca, 2020) e «formanti» (Dardano, 2009).

Il mio intento di oggi è soffermarmi non già su questi casi assodati, ma su alcuni casi problematici, sottogruppi di parole che ultimamente hanno ricevuto nuova attenzione dagli studiosi del settore. Si tratta di sequenze che condividono con le parole composte il fatto di essere degli agglomerati di più parole autonome aventi un significato unitario (ne fa un'utile rassegna Masini, 2012). Alcuni esempi sono le parole elencate in (1) e (2) che appartengono a varie categorie lessicali: vi sono elencati nomi (N), verbi (V), aggettivi (Agg), avverbi (Avv). Chiameremo queste sequenze semplicemente parole composte? O hanno dei comportamenti tali per cui è giusto distinguerle e dare loro un nome specifico?
(1) ferro da stiro ${ }_{\mathrm{N}}$ senza arte né parte ${ }_{\mathrm{Agg}}$ andare in porto ${ }_{\mathrm{v}}$ a tempo pieno $_{\mathrm{Avv}}$
(2) cartone animato $_{\mathrm{N}}$ alla mano $_{\mathrm{Agg}}$ stare fresco $\mathrm{V}$ a regime $_{\mathrm{Avv}}$

\subsection{Parole composte e parole polirematiche}

Negli studi di morfologia lessicale si distingue tra parole composte e parole polirematiche. In realtà il termine 'polirematico'2, di chiara origine greca, significa letteralmente 'composto da più parole'. Ma siccome anche i composti sono formati da più parole, qual è esattamente il confine tra i due sottogruppi? Perché i morfologi hanno sentito il bisogno di suddividere e diversificare? È certo

2 'Polirematico' si oppone a 'monorematico', che significa 'formato da una sola parola'. 
che alcuni repertori lessicografici contengono liste di parole polirematiche: così De Mauro et al. (1993) e De Mauro (1999-2000). È giusto accogliere anche nella scuola questa distinzione?

Nel Quadro di riferimento delle prove Invalsi di italiano (2018) la scelta è fatta: nell'elencare gli indici relativi ai diversi ambiti grammaticali su cui vertono le prove, alla voce Formazione delle parole compaiono i seguenti indici: «Parole semplici e parole complesse; parole di base e parole derivate; parole alterate; parole composte; parole polirematiche (ferro da stiro, asilo nido)» (p. 12). ̇̀ significativo che l'Istituto accompagni con esempi solo il termine ritenuto probabilmente meno noto o meno condiviso dai docenti. E si noti che gli esempi sono due: una parola a tre membri, una a due, come nei nostri due gruppi (1) e (2).

Vediamo allora le ragioni dei morfologi, o almeno di qualcuno di essi. Tutti individuano la categoria delle parole polirematiche, chiamate anche 'parole sintagmatiche', 'composti sintagmatici', 'unità lessicali superiori', 'espressioni multiparola'. Ma mentre alcuni inseriscono senz’altro il paragrafo sulle parole polirematiche all'interno del capitolo sulla composizione (Voghera, 2004; Dardano, 2009; Lo Duca, 2020), altri preferiscono trattarne in uno spazio dedicato (ad es. Micheli, 2020), presentando le polirematiche come delle parole a metà strada tra i composti e i sintagmi veri e propri (Scalise \& Bisetto, 2008, p. 209), di cui spesso condividono la struttura: come nel caso del sintagma casa di campagna e della polirematica carta di credito. I criteri per distinguere il primo dalla seconda sono, tra gli altri:

1. la coesione interna, cioè l'inseribilità o meno di materiale lessicale tra i costituenti, sempre possibile nei sintagmi (una casa bellissima di campagna), spesso impossibile o almeno innaturale nelle polirematiche ( ${ }^{\star}$ una carta scaduta di credito vs. una carta di credito scaduta);

2. la sostituibilità di uno dei due membri con altre parole, purché ovviamente coerenti sul piano semantico, sempre possibile nei sintagmi (villa di campagna, casa di periferia), per lo più impossibile nelle polirematiche $\left({ }^{\star} d o c u-\right.$ mento di credito, * carta di acquisto). 
Si tratta di criteri di massima, che generalmente funzionano anche se non in tutti i casi con la stessa forza e con la stessa regolarità.

Per quanto riguarda invece la distinzione tra parole composte e parole polirematiche, quali sono i criteri su cui si basano i morfologi per distinguere i due sottogruppi? I quali peraltro condividono molte importanti proprietà: hanno un significato unitario, riconosciuto come tale dai parlanti; sono generalmente dotati di coesione interna, quindi non tollerano inserzioni di elementi estranei tra i costituenti; non ammettono di solito la sostituzione di uno dei costituenti. In che cosa si differenziano, dunque?

Bisetto (2004, p. 36) suggerisce dei criteri che, spogliati dei loro risvolti più tecnici, si potrebbero facilmente adottare anche a scuola. Il primo criterio è formale: le parole composte sono formate da due parole autonome, le polirematiche da due, tre o più. Quindi le parole elencate in (1) sono tutte polirematiche, perché formate da tre o più parole. Le parole elencate in (2), invece, sono a due costituenti, e potrebbero essere sia composte che polirematiche. Come faremo a distinguere?

A questo punto entra in gioco il secondo criterio, che è di tipo semantico: il significato delle parole composte è, come abbiamo già detto, composizionale, dato cioè dalla somma dei significati dei due elementi costitutivi. Nel caso però delle formazioni in (2) il significato non è composizionale: un cartone animato non è una 'carta spessa e pesante dotata di movimento' ma 'un tipo di film costruito secondo una tecnica particolare'; e dire di qualcuno che è alla mano non significa dire che è 'vicino alla mano' (di chi?), ma piuttosto che è 'semplice, cortese, disponibile'; stare fresco non significa 'stare in una temperatura fresca' ma piuttosto 'trovarsi in una brutta situazione'; infine a regime non richiama nessuno dei molti significati di regime ma indica 'il momento in cui qualcosa raggiunge il suo pieno sviluppo'. Chiameremo dunque polirematiche anche questi agglomerati a due costituenti di significato non composizionale.

Sulla base di questo criterio le parole in (3) sono parole composte, perché hanno un significato composizionale; le parole in (4) sono parole polirematiche, con significato idiomatico, non composizionale. 
(3) treno merci vagone ristorante guerra lampo ('guerra rapida come un lampo') cassa integrazione diritto dovere
(4) asilo nido anima gemella passo falso giornale radio sotto torchio

Non viene invece mai invocato il criterio assunto dalle poche grammatiche scolastiche che parlano di polirematiche - o più spesso di 'unità lessicali superiori' o 'unità lessicali complesse' - vale a dire la scrittura univerbata dei componenti nelle parole composte e, al contrario, la scrittura separata nelle polirematiche. Secondo questo criterio tutte le parole in (3) e in (4) sarebbero polirematiche, ma, del tutto incoerentemente, spesso questo o quel membro compare nelle liste delle parole composte. La verità è che nella resa grafica delle parole composte non esiste una norma condivisa, per cui gli stessi dizionari registrano capocarceriere e capo carceriere o acquasanta e acqua santa, e non sono rari i casi in cui compare anche il trattino di separazione tra i due costituenti, come in sala-computer o diritto-dovere, senza che sia possibile risalire a una qualche regolarità di comportamento (Micheli, 2020, p. 111; Lo Duca, 2020, p. 85). Ma, indipendente dal modo in cui sono scritti, questi esempi, tutti a due costituenti e di significato composizionale, sono dai morfologi etichettati come parole composte.

E tuttavia, il criterio semantico che ci aiuta a distinguere le parole composte da quelle polirematiche a due costituenti è, appunto, un criterio semantico, che interroga la sensibilità del parlante di oggi, ma non tiene conto della storia e dell'evoluzione delle parole. Ci sono formazioni che, nate con significato composizionale, si sono poi evolute nel tempo acquisendo significati metaforici, e le due letture spesso convivono nelle stesse formazioni: come nel caso di alta tensione ('tensione elettrica superiore a 30.000 volt' e 'stato ansioso, condizione di angoscia e forte apprensione') o di bandiera bianca ('bandiera bianca usata in guerra in segno di resa' e 'rinuncia a perseguire un piano, un progetto'). Come etichetteremo queste formazioni, composte o polirematiche?

La risposta non è molto importante, e anche gli studiosi del settore ammettono che «il confine tra di essi è spesso difficile da tracciare» (Micheli, 2020, p. 
194). Ma se con i nostri studenti saremo giunti fin qui nel ragionamento potremo dirci soddisfatti, avremo già ottenuto lo scopo che qualsiasi attività riflessiva sulla lingua deve proporsi: che è non già quello di mettere le etichette giuste, ma di acquisire i mezzi per mettere ordine tra i fatti linguistici, esplorarne le proprietà e chiarire i concetti. Ragionare sul significato letterale o figurato delle formazioni multilessicali, risalire alla loro storia consultando un dizionario cartaceo o on-line, questi sono sicuramente esercizi utili sia ad ampliare il bagaglio lessicale dei giovani, sia ad affinare la loro sensibilità lessicale: obiettivi, questi sì, di tutto rispetto, da perseguire dal primo all'ultimo anno di scuola, università compresa.

Rimangono fuori da questa bipartizione alcune sottocategorie che a volte trovano posto nella trattatistica scolastica: come ad esempio le 'parole-frase' e i 'conglomerati's. Quanto alle prime, questa categoria compare almeno in due grammatiche scolastiche fra le più adottate: Zordan (2019) e Sensini (2018). Zordan dà come esempi di parole-frase cerniera lampo e gonna pantalone, cui accompagna questa definizione: «sono espressioni che nascono dall'accostamento di due parole le quali rimangono separate l'una dall'altra, cioè non si fondono per dar vita a un'unica parola, come invece avviene nelle parole composte propriamente dette». Criterio assolutamente fallace, come abbiamo già scritto e come tutti i morfologi non mancano di notare. Anche un sito frequentato, temo, da molti insegnanti (lagrammaticaitaliana.it) dà la stessa definizione e alcuni esempi (cane poliziotto, uomo ragno, romanzo fiume).

Da tutti questi esempi ricavo che vengono denominati parole-frase quei composti (che in letteratura vengono da qualcuno chiamati 'appositivi' o 'giustapposti') con struttura N-N, in cui il secondo elemento è un nome che fa da modificatore del nome testa del composto, e richiede una interpretazione figurata: così una cerniera lampo è 'una cerniera che si chiude con la stessa velocità di un lampo' e un uomo ragno è un 'uomo che si arrampica come un ragno'. È certamente un bene riflettere sulle particolari proprietà semantiche e sintattiche di queste parole, ma nei libri citati non lo si fa, e ci si limita a dire che si tratta di parole «che riassumono, sostituendola, un’intera frase» (Zordan, 2019, p. 799).

3 Devo questi dati sulle grammatiche scolastiche a Maria Cristina Peccianti, che pubblicamente ringrazio. 
Ma questo è un criterio vaghissimo, che potrebbe applicarsi a migliaia di parole, e non solo composte e derivate: così ad esempio il portasci (parola composta) è un 'supporto per il trasporto di sci che si applica sul tetto o sul retro della vettura'; il contatore (parola derivata) è un 'apparecchio che segnala il numero di operazioni effettuate da una macchina o dispositivo'; il divano (parola semplice) è un 'ampio sedile imbottito, destinato a più persone'. Tutto questo per dire che non vedo la necessità di accorpare parole come romanzo fiume o gonna pantalone in una sottocategoria dedicata. Basterà accogliere la sistemazione adottata nei trattati di morfologia lessicale, che elencano queste formazioni tra le parole composte del tipo N-N.

Anche i 'conglomerati' sono una sottocategoria di cui potremmo facilmente fare a meno. Il vocabolario Treccani in rete ne dà la seguente definizione: «unità lessicale composta, risultante dalla fusione di più parole, per lo più due verbi, cristallizzati in una sola locuzione invariabile, usata come s.m.». Gli esempi riportati sono saliscendi e tiremmolla, coerenti con la definizione. Sia la grammatica di Sensini (2018, p. 176) che quella di Serianni, Della Valle e Patota (2017, p. 99) parlano di conglomerati: ma quest'ultima elenca fuggifuggi e dormiveglia sia tra $i$ «composti veri e propri» sia tra $i$ «conglomerati». Nella riflessione moderna sulla composizione queste formazioni (che sono pochissime, anche perché il procedimento non è più produttivo in italiano) vengono molto più semplicemente etichettate come parole composte del tipo V+V. È del tutto inutile appesantire la memoria degli studenti con una etichetta così poco rappresentativa ${ }^{4}$.

\subsection{Verbi sintagmatici, verbi polirematici o verbi composti?}

Sono i verbi elencati in (5), studiati in italiano per la prima volta in modo mirato da Simone (1997, p. 157) che li ha denominati 'verbi sintagmatici', sulla falsariga dell'inglese phrasal verbs, cui assomigliano molto.

4 Qui sarebbe da aprire una parentesi, su questo eccesso di zelo di cui danno spesso prova le grammatiche scolastiche, particolarmente abili a denominare con termini appositi gruppi, sotto-gruppi, sotto-sotto-gruppi. Ma mi riservo di tornare sull'argomento in una prossima occasione. 
(5) andar(e) via/su/giù

buttar(e) via/giù

mettere sotto/giù

tirare su

Sono formazioni molto diffuse in vari dialetti italiani (Iacobini \& Masini, 2009) e nel parlato, e sembra che il loro numero sia in crescita. Come si vede dagli esempi, si tratta di verbi a due costituenti, un verbo per lo più di movimento e un avverbio di tipo locativo che specifica la direzione del movimento (andare fuori, buttare via), con significato composizionale. Tuttavia a volte l'avverbio è pleonastico, nel senso che si limita a rafforzare il significato del verbo (come in uscire fuori, salire su, buttare giù), mentre non sono rari i casi in cui il significato non è più composizionale, ma metaforico, in tutto o in parte: come in fare fuori nel senso di 'uccidere', 'eliminare', o andare dietro a qualcuno nel senso di 'corteggiare', o mettere sotto nel senso di 'investire' (detto di un autoveicolo).

Una prova del fatto che si tratti di agglomerati unitari è data dal troncamento che il costituente verbale a volte subisce (e le forme tronche sono forse addirittura più frequenti delle corrispondenti forme piene): scrivendo, e soprattutto parlando, usiamo dire andar via, buttar giù, salir su, metter via, e l'insieme tende a costituire un'unica parola fonologica con accento principale sul costituente avverbiale (Simone, 1997, p. 167).

Una ulteriore prova è data dalla coesione interna di queste formazioni, che sembrano condividere con le parole composte e con le parole polirematiche la proprietà di non poter essere interrotte da altro materiale lessicale: così buttare giù la pasta suona molto più naturale di buttare la pasta giù, e andare via di corsa pare meglio formato di andare di corsa via. Sono tuttavia agglomerati che rispondono al test in modo non sempre coerente, probabilmente in via di lessicalizzazione specie in alcuni usi ricorrenti, che hanno spesso sviluppato dei significati metaforici: come ad esempio tirare su un bambino, nel significato di 'allevare, educare un bambino' o andare dietro a qualcuno nel senso di 'corteggiare'. Il problema è che queste stesse sequenze possono essere usate anche nel loro significato letterale, composizionale, e in questo caso i due costituenti possono essere interrotti, come in ti dispiacerebbe tirare il bambino/il pacco su?; 
il mio gatto va sempre dietro ai topi. In questi casi i complessi verbali in discussione vanno interpretati come dei sintagmi verbali.

Lo statuto incerto di queste formazioni è evidenziato anche dalla diversa terminologia con cui vengono designate: la dicitura di ‘verbi sintagmatici’ proposta, come abbiamo già detto, da Simone (1997), è stata adottata da molti altri studiosi (tra cui Iacobini \& Masini, 2009; Jansen, 2011; Masini, 2012); altri li inseriscono nel gruppo delle parole composte (Lo Duca, 2020, p. 97) o polirematiche (Voghera, 2004, pp. 65-66; Micheli, 2020, pp. 194-195), a seconda che accentuino la composizionalità (composti) o la non composizionalità (polirematiche) del significato. Ma anche in questo caso l'etichetta non è importante. È importante invece che docenti e studenti abbiano chiare le particolari caratteristiche di questo sottogruppo di verbi (di cui c'è poca o nessuna traccia nelle grammatiche scolastiche) e le ragioni della diversità delle denominazioni, da cui non dovrebbero lasciarsi intimidire, scegliendo quella che pare più semplice, o più coerente, o più condivisa.

\section{Conversione}

Non so se e quanto si parli di conversione nei libri per le scuole ${ }^{5}$, ammetto che non ho condotto sul tema nessuna ricerca specifica. Ma so per certo che alcune parole formatesi per conversione creano difficoltà di analisi in molti docenti, non aiutati in questo dai libri in adozione nelle loro classi. Proverò dunque a chiarire il fenomeno generale, per passare poi a esaminare alcuni casi particolarmente spinosi.

Conversione indica «il fenomeno per cui una parola 'si converte' da una categoria lessicale all’altra senza aggiunta di morfemi derivazionali, assumendo, in questo passaggio, le caratteristiche prototipiche della categoria di arrivo» (Lo Duca, 2020, p. 105). La conversione è, dunque, «un processo che possiamo definire di transcategorizzazione» (Scalise \& Bisetto, 2008, p. 198). Un esempio classico di conversione in italiano è costituito dai casi in cui degli aggettivi diventano nomi senza l'aggiunta di suffissi derivazionali. Il fenomeno, che in questo caso si risolve in un processo di nominalizzazione, riguarda in modo parti-

5 Ma sempre Maria Cristina Peccianti mi dice che non ne ha mai trovato traccia. 
colare alcune sottocategorie di aggettivi: ad esempio gli aggettivi etnici (i vini francesi, le canzoni napoletane) possono essere usati come nomi, a indicare gli abitanti del luogo (i/le francesi, un/una francese; le napoletane, una napoletana) e anche la lingua, questa volta sempre col genere maschile e l'articolo determinativo (il francese, il napoletano); gli aggettivi di colore, usati come nomi, possono designare il colore (mi piace molto il verde), gli aderenti a un gruppo o a un'ideologia (i verdi, i rossi) o una squadra sportiva (gli azzurri). Lo stesso dicasi di casi come il bello, l'utile, che designano nomi di qualità, resi più spesso attraverso dei procedimenti derivativi (rispettivamente bell-ezza, util-ità).

In morfologia lessicale il fenomeno della conversione viene anche chiamato, soprattutto da alcuni studiosi di scuola generativa, 'derivazione zero', o 'suffissazione zero' (Salvi \& Vanelli, 2004; Scalise, 1995), denominazione che richiama una sorta di «suffisso “astratto”, non realizzato foneticamente» (Salvi \& Vanelli, 2004, p. 335) e reso col simbolo Ø, «a sottolineare il fatto che il cambio categoriale comporta sempre l'aggiunta di un affisso, il quale però può, a certe condizioni, risultare fonologicamente vuoto» (Lo Duca, 2020, p. 105). Ad essere ancora più precisi, si potrebbe anche argomentare a favore di una qual certa differenza tra i due termini, e di conseguenza tra i diversi fenomeni, come fanno ad esempio Scalise e Bisetto (2008, pp. 197-199), ma non li seguiremo su questa strada.

Come la maggior parte degli autori (ad esempio Thornton, 2004; Grossmann, 2004; Iacobini, 2010b), considererò sinonimi i due termini e userò 'conversione', che trovo più neutro rispetto alle diverse teorie linguistiche, limitandosi il termine a descrivere l'operazione superficiale di passaggio, di trasferimento o, appunto, conversione di una parola da una categoria lessicale ad un'altra.

\subsection{Conversione o derivazione? Da nome a verbo e da verbo a nome}

Normalmente nei trattati di morfologia lessicale relativi all'italiano (Grossmann \& Rainer, 2004; Lo Duca, 2020; Micheli, 2020) tra i primi casi di conversione viene elencato un sottogruppo di parole esemplificato da sale $\rightarrow$ sal-are, squama $\rightarrow$ squam-are, zucchero $\rightarrow$ zuccher-are, con base nominale, o da calmo $\rightarrow$ calm-are, preciso $\rightarrow$ precis-are, stanco $\rightarrow$ stanc-are con base aggettivale. Viene ricordato anche qualche raro caso di formazioni verbali da basi avverbiali, come addosso 
$\rightarrow$ addoss-are, attraverso $\rightarrow$ attravers-are. Perché questi casi sono considerati forme di conversione e non di derivazione? Non si era detto che nella conversione la parola di base cambia categoria lessicale senza l'aggiunta di morfemi derivazionali?

In realtà -are, che abbiamo aggiunto alle parole in esempio, non è un morfema derivazionale (come -eggiare in caldo $\rightarrow$ cald-eggiare, o -ificare in vino $\rightarrow$ vin-ificare, o -izzare in canale $\rightarrow$ canal-izzare). È un morfema flessivo, e l'italiano, si sa, è una lingua flessiva, dunque un nome non può diventare verbo senza assumere necessariamente le marche morfologiche del verbo. In inglese, che è una lingua con scarsa morfologia, la conversione è un fenomeno più trasparente: stop vuol dire 'fermata' ma anche 'fermare', come water vuol dire 'acqua' e anche 'dare acqua, innaffiare'. Ma in italiano se da un nome si forma un verbo è di tutta evidenza che il verbo dovrà assumere il morfema flessivo dei verbi: nei nostri esempi le parole di base, rispettivamente sale, squama, zucchero, calmo, preciso, stanco perdono il morfema flessivo finale (che determina la classe flessiva del nome o dell'aggettivo), e al suo posto assumono la vocale tematica (in questo caso - $a$-) e il morfema dell'infinito dei verbi, quindi sal-a-re, squam-a-re o calm-a-re, stanc-a-re, allineandosi così ai verbi della I coniugazione ${ }^{6}$.

Ma sorge immediato un problema: che cosa ci fa dire, negli esempi fatti, che il processo non sia avvenuto al contrario? Che cioè dai verbi salare, squamare, stancare non si siano formati, per conversione, sale, squama e stanco? Il ragionamento fatto sopra potrebbe adattarsi molto bene anche a questa possibilità: dei verbi perderebbero il morfema flessivo della I coniugazione per adattarsi alle caratteristiche morfologiche delle categorie di arrivo: un nome maschile in $-e$, plurale in $-i$, per sale; un nome femminile in $-a$, plurale in -e, per squama; un aggettivo della I classe in -o/-i, - a/-e per stanco e così via. E dunque come si fa in questi casi a capire la direzione del cambiamento? Dai nomi ai verbi, o dai verbi ai nomi?

6 Risultano piuttosto rare le formazioni che assumono la vocale tematica $-i-$, dando origine a verbi della III coniugazione (come in favor-i-re, snell-i-re), procedimento che Grossmann (2004, p. 534) giudica oggi non più produttivo. 
Tanto più che per un altro sottogruppo di parole, non meno numeroso e apparentemente simile, l'analisi che viene fatta è opposta. Per accusa, proroga, conquista e per arrivo, spaccio, salto, nomi rispettivamente femminili e maschili, tutti morfologicamente relati a verbi (accusare, prorogare, conquistare e arrivare, spacciare, saltare), l'analisi di gran lunga prevalente tra i morfologi è la seguente: questi nomi si sono formati, per conversione, dai rispettivi verbi, quindi accusare $\rightarrow$ accusa, come saltare $\rightarrow$ salto. Che cosa fa arrivare a questa conclusione?

Una via per controllare la direzione del cambiamento potrebbe essere quella di consultare, per ognuna di queste parole, un buon dizionario etimologico, per tentare di stabilire quale parola sia attestata per prima, il nome o il verbo, e stabilire per questa via la direzione del cambiamento. Ma si tratta di una via complessa e poco sicura, che in qualche caso ci direbbe non già la parola apparsa per prima, ma la parola di cui la prima attestazione nota è più antica. Nuove esplorazioni potrebbero sempre rimettere in discussione conclusioni che parevano assodate.

La soluzione al problema va dunque cercata altrove. Proviamo a ragionare sull'esito semantico di tutte queste formazioni. A differenza di sale, squama, zucchero che designano ciò che potremmo chiamare genericamente 'oggetti' o 'sostanze', tutti i nomi che vengono dati come formatisi dai rispettivi verbi di base per conversione sono nomi d'azione: accusa e proroga sono rispettivamente 'l'atto dell'accusare' e 'l'atto del prorogare', così come arrivo e spaccio sono 'l'atto dell'arrivare' e 'l'atto dello spacciare'. Quello della formazione di nomi d'azione da verbi è peraltro una eventualità tutt’altro che rara nella lingua italiana (e non solo), e non si limita alla conversione, ma riguarda in modo assai più rilevante - per numerosità dei procedimenti e delle formazioni - la derivazione. Ci sono molti suffissi che formano nomi di azione da verbi: dai più produttivi -zione (segrega-zione, trasforma-zione) e -mento (abbina-mento, possedi-mento), a -tura (pota-tura, spremi-tura), -aggio (lav-aggio, pest-aggio), -anza (milit-anza, toller-anza), -io (balbett-io, miagol-io) ed altri. In tutti i casi i nomi di azione derivati da verbi hanno la stessa parafrasi, 'atto di V', quindi 'atto del segregare', 'atto dell'abbinare', 'atto del potare’ e così via. Grazie alla presenza del suffisso, in questi casi non abbiamo dubbi: la direzione del cambiamento è chiara, e va dal verbo al nome. 
Ma allora, se i nomi di azione si formano a partire dal verbo corrispondente, non c'è motivo di credere che nei casi di conversione le cose stiano diversamente. Basterà a questo punto riflettere sulle trasformazioni formali subite da questi tre diversi gruppi di parole:

A. arrivare $\rightarrow$ arrivo, 'atto dell'arrivare'

saltar $\rightarrow$ salto, 'atto del saltare'

acquistare $\rightarrow$ acquisto, 'atto dell'acquistare'

B. conquistare $\rightarrow$ conquista, 'atto del conquistare'

ricercare $\rightarrow$ ricerca, 'atto del ricercare'

bonificare $\rightarrow$ bonifica, 'atto del bonificare'

C. promettere $\rightarrow$ promesso $\rightarrow$ promess a, 'atto del promettere'

bere $\rightarrow$ bevuto $\rightarrow$ bevuta, 'atto del bere'

schiarire $\rightarrow$ schiarito $\rightarrow$ schiarita 'atto dello schiarire'

Il gruppo A esemplifica nomi formatisi dalla radice del verbo, rispettivamente arriv-, salt-, acquist- e assunzione del genere maschile e della classe flessiva dei nomi in - $o$, plurale in - $i$. I nomi in B si sono formati dal tema del verbo, quindi dalla radice + la vocale tematica in - $a$ (da notare che le formazioni di questo tipo hanno come base verbi della I coniugazione), il che determina l'assunzione del genere femminile e della classe flessiva dei nomi in - $a$, plurale in -e. I nomi del gruppo $\mathrm{C}$ si sono formati non già dal tema dell'infinito dei rispettivi verbi di base, ma dal tema del participio passato, con assunzione del genere femminile e della classe flessiva dei nomi in - $a$, plurale in - $e$. Questo terzo sottogruppo è molto produttivo, e comprende anche formazioni da participi passati irregolari (correre $\rightarrow$ corso $\rightarrow$ corsa, scrivere $\rightarrow$ scritto $\rightarrow$ scritta, leggere $\rightarrow$ letto $\rightarrow$ letta $)^{7}$.

7 In realtà, se consultassimo un dizionario etimologico, potremmo scoprire che alcune di queste formazioni non si sono formate in italiano ma sono di origine latina, e sono passate in italiano a processo di formazione già avvenuto. Questo non inficia il nostro ragionamento. In morfologia lessicale è buona regola assumere «una prospettiva sincronica, che guarda all’oggi con la sensibilità del parlante di oggi» (Lo Duca, 2020, p. 14). Solo di fronte a casi formalmente o semanticamente irregolari sarà utile ricorrere alla storia. Ad esempio: parole come mangione, borbottone, arraffone si sono formate regolarmente dai rispettivi verbi di base (mangi-one, borbott-one, arraff-one) ed esibiscono una semantica trasparente e regolare, rispettivamente 'persona che mangia tanto, in modo smodato', 'persona che borbotta continua- 
E qui mi fermo, anche se il capitolo della conversione non si esaurisce a questi pochi casi (per una presentazione sistematica ma non troppo tecnica della materia rimando a Lo Duca, 2020, pp. 105-117). Prima di concludere, vorrei però precisare un ultimo punto: ho chiamato nomi di azione i nomi formatisi da verbi per conversione, assimilandoli ai nomi di azione formatisi da verbi per derivazione. Tuttavia qualcuno potrebbe nutrire dei dubbi sulla semantica di alcuni degli esempi fatti. In realtà molti dei cosiddetti nomi di azione, lo sono in certi contesti ma non in altri. Prendiamo due esempi, due nomi formatisi da verbi, l'uno per derivazione, l'altro per conversione. Pubblicazione, formatosi per derivazione da pubblicare+-zione, indica l'atto del pubblicare in la pubblicazione del romanzo è stata vietata, il risultato del pubblicare in il candidato ha presentato le sue pubblicazioni. Anche parcheggio, nome d'azione formatosi per conversione da parcheggiare, designa l'atto del parcheggiare in di fronte a casa mia il parcheggio è vietato, un nome di luogo in di fronte a casa mia c'è un parcheggio. Anzi, se consulto un dizionario, la prima accezione che viene data di parcheggio è proprio la seconda ('area riservata alla sosta dei veicoli').

I due esempi ci ricordano che, come ci avvertono tutti gli studiosi del settore (Gaeta, 2002, 2004; Fiorentino, 2010), i nomi di azione, tendenzialmente astratti, hanno spesso subito, e continuano a subire, slittamenti di significato potendo designare anche oggetti più concreti: come i risultati dell'azione, ad esempio pubblicazione, già visto, o guadagno in i suoi guadagni sono modesti; il luogo in cui si realizza l'azione, come parcheggio o accampamento, quest'ultimo derivato; lo strumento con cui si realizza l'azione, ad esempio cambio in il cambio di quest'auto non funziona.

\section{Parasintesi}

È un fenomeno forse marginale, ma vale la pena di soffermarvisi brevemente perché interessa parole molto frequenti nella lingua, e il mancato riconosci-

mente', 'persona che arraffa più che può'. Anche dormiglione significa 'persona che dorme molto, in modo eccessivo', quindi la semantica è coerente con quella dei deverbali in -one. Ma sul piano formale non ci siamo: dal verbo dormire avremmo dovuto avere ${ }^{*}$ dorm-one, non dormigli-one. A questo punto vale la pena di consultare un dizionario etimologico, il quale ci dirà subito che dormigli-one si è formato da dormigli-are, forma arcaica di dormire. Tutto ciò per dire che quando non riusciamo a spiegarci certe anomalie, conviene esplorare la storia delle parole e ripercorrerne l'evoluzione. 
mento di questo procedimento genera incertezze di analisi e conseguentemente errori. È una categoria individuata relativamente di recente negli studi di morfologia lessicale, e ad esempio ancora nella grammatica di Fornaciari (19318, p. 118) alcuni parasintetici (accoppiare, accavallare) vengono tranquillamente elencati tra i verbi prefissati, e questa sistemazione perdura ancora oggi in molti libri destinati alle scuole (e non solo). Ma se fossero dei verbi prefissati, quali sarebbero i verbi cui il prefisso si attacca, visto che ${ }^{\star}$ coppiare ${ }^{\star}{ }^{\star}$ cavallare non esistono?

In realtà la parasintesi è un procedimento complesso che comporta un doppio movimento: a partire da nomi e da aggettivi si formano verbi per prefissazione e per conversione, e questi due procedimenti avvengono contemporaneamente. Ad esempio il verbo in-caten-are si è formato da catena grazie alla contemporanea assunzione del prefisso in- e la conversione del nome di base in verbo, e quindi l'assunzione del suffisso flessivo dei verbi della prima coniugazione. Lo stesso potremmo dire per im-bottigliare-are o ab-botton-are, denominali, e per ar-ricch-ire o s-vil-ire, deaggettivali, che assumono invece il suffisso flessivo dei verbi della terza coniugazione.

Come ho già detto, formazioni di questo tipo vengono spesso etichettate tra le parole prefissate, o anche tra le parole suffissate, a seconda che si dia rilevanza al prefisso, o che si interpreti erroneamente la conversione come un fatto di suffissazione. In realtà non esistono né i verbi * bottigliare, ${ }^{*}$ bottonare, ${ }^{*}$ ricchire, ${ }^{*}$ vilire (se esistessero potremmo dire che im-bottigliare, ab-bottonare, ar-ricchire, s-vilire si sono formati per prefissazione); né i nomi *imbottiglia/e, ${ }^{*}$ abbottona/e, o gli aggettivi ${ }^{*}$ arricco/a/hi/he, ${ }^{*}$ svilo/i/a/e (se esistessero potremmo parlare di conversione in verbi di nomi e di aggettivi). Quindi dobbiamo supporre che questi verbi si siano formati attraverso un doppio procedimento simultaneo, di prefissazione e di conversione, e quindi è giusto chiamarli con un nome dedicato.

Una questione spinosa per un non linguista, e che potrebbe confondere gli studenti, è la forma dei prefissi: se guardiamo agli esempi, dobbiamo supporre che i segmenti in-, im-, $a b-$, $a r-$, di- siano tutti dei prefissi? Alcuni di questi effettivamente lo sono (in-, s-), e li ritroviamo in questa stessa forma in centinaia di parole dell'italiano; tutti gli altri, invece, sono delle varianti (la parola tecnica è 
'allomorfi') di suffissi molto ben conosciuti quali, appunto, in- e ad-. Dunque in im-bottigliare il prefisso in- diventa im- perché precede una parola che inizia per una occlusiva bilabiale, e lo stesso accade in molti altri parasintetici (im-beccare, im-bocc-are, im-pacc-are, im-pallid-ire), che si uniformano ad una regola molto generale dell'italiano. Per quanto riguarda invece il prefisso ad-, esso rimane inalterato solo se la parola di base inizia per vocale (ad-agi-are, ad-esc- $a$ re). In tutti gli altri casi subisce un processo di assimilazione alla consonante iniziale della parola di base: quindi non solo $a(d) b$-botton-are e $a(d) r$-ricch-ire, ma anche $a(d)$ l-legger-ire, $a(d) f$-fatic-are, $a(d) c$-cald-are, $a(d) g$-grav-are e così via.

\section{Conclusioni}

Spero di essere riuscita nell'intento che mi ero prefissata: dimostrare che la terminologia grammaticale non è una variabile di poco conto in un programma ben meditato di riflessione sulla lingua. Per un docente di italiano usare i termini tradizionali può essere una scelta comoda, ma a condizione di avere ben chiari in testa gli oggetti linguistici cui quei termini si riferiscono. Il problema, per un docente poco esperto di grammatica moderna, è che i termini della tradizione a volte sono stati abbandonati, più spesso sono stati ridefiniti dalla ricerca grammaticale di questi ultimi decenni, e può accadere che termini tradizionali come, ad esempio, parola derivata o parola composta, abbiano subito dei rimaneggiamenti importanti che ne hanno modificato, più o meno profondamente, il raggio di applicazione.

In quest’opera di revisione e scandaglio, che i mezzi tecnologici moderni hanno reso infinitamente più facile proprio nel campo dello studio del lessico, l'individuazione di proprietà e comportamenti regolari da parte di piccoli o grandi sottogruppi di parole ha spesso reso inevitabile l'adozione di un termine dedicato, poco noto al grande pubblico. Di questi nuovi termini si sono talvolta impadroniti i libri di testo per le scuole di ogni ordine e grado, che li hanno usati in modo a volte maldestro, limitandosi per lo più ad accoglierli come sinonimi di termini tradizionali, con cui vengono fatti convivere nella stessa pagina (clamoroso e ben noto il caso di 'espansione', usato senza ulteriori specificazioni come sinonimo moderno del più generico 'complemento'). Vorrei dire agli inse- 
gnanti di vigilare: non sempre il termine moderno rende più attuale e in linea con la ricerca il loro insegnamento. Dietro un termine nuovo c'è per lo più un oggetto linguistico spesso già noto, ma diversamente analizzato e accorpato, e quindi etichettato. Cambiargli nome senza cambiare analisi e prospettive di solito non funziona, e ha purtroppo l'esito di aumentare la confusione, già grande sotto il cielo grammaticale.

\section{Bibliografia}

Colombo, A. (2009). Il pronome determinativo. Studi linguistici italiani. XXXV (II)/2009, 285-293.

Dardano, M. (2009). Costruire parole. La morfologia derivativa dell'italiano. Bologna: Il Mulino.

De Mauro, T., Mancini, F., Vedovelli, M., \& Voghera, M. (1993). LIP. Lessico di frequenza dell'italiano parlato. Milano: Etaslibri.

De Mauro, T. (1999-2000). GRADIT. Grande Dizionario italiano dell'uso (6 + 1 voll.). Torino: Utet.

Fiorentino, G. (2010). Azione, nomi di. In R. Simone, G. Berruto \& P. D’Achille (a cura di), Enciclopedia dell'Italiano (vol. I, pp. 135-138). Roma: Istituto dell'Enciclopedia italiana. https://www.treccani.it/enciclopedia/nomi-di-azione \%28Enciclopedia-dell\%27Italiano\%29/

Gaeta, L. (2002). Quando i verbi compaiono come nomi: un saggio di morfologia naturale. Milano: Franco Angeli.

Gaeta, L. (2004). Nomi d'azione. In M. Grossmann \& F. Rainer (a cura di), La formazione delle parole in italiano (pp. 314-351). Tübingen: Max Niemeyer.

Grossmann, M. (2004). Conversione in verbi. In M. Grossmann \& F. Rainer (a cura di), La formazione delle parole in italiano (pp. 534-546). Tübingen: Max Niemeyer.

Grossmann, M., \& Rainer, F. (a cura di) (2004), La formazione delle parole in italiano. Tübingen: Max Niemeyer. 
Iacobini, C. (2004). Composizione con elementi neoclassici. In M. Grossmann \& F. Rainer (a cura di), La formazione delle parole in italiano (pp. 69-95). Tübingen: Max Niemeyer.

Iacobini, C. (2010a). Elementi formativi. In R. Simone, G. Berruto \& P. D'Achille (a cura di), Enciclopedia dell'Italiano (vol. I, pp. 416-418). Roma: Istituto dell'Enciclopedia italiana. https://www.treccani.it/enciclopedia/elementi-formativi_\%28Enciclopedia-dell\%27Italiano\%29/

Iacobini, C. (2010b). Conversione. In R. Simone, G. Berruto \& P. D’Achille (a cura di), Enciclopedia dell'Italiano (vol. I, pp. 292-294). Roma: Istituto dell'Enciclopedia italiana. https://www.treccani.it/enciclopedia/conversione \%28Enciclopedia-dell\%27Italiano\%29/

Iacobini, C., \& Masini, F. (2009). I verbi sintagmatici dell'italiano tra innovazione e persistenza: il ruolo dei dialetti. In A. Cardinaletti \& N. Munaro (a cura di), Italiano, italiani regionali e dialetti (pp. 115-135). Milano: Franco Angeli.

Invalsi (2013). Quadro di riferimento della prova di italiano. https://invalsi-area prove.cineca.it/docs/file/QdR_Italiano_Obbligo_Istruzione.pdf

Jansen, H. (2011). Sintagmatici, verbi. In R. Simone, G. Berruto \& P. D’Achille (a cura di), Enciclopedia dell'Italiano (vol. II, pp. 1348-1352). Roma: Istituto dell'Enciclopedia italiana.

Lo Duca, M. G. (1990). Creatività e regole. Studio sull'acquisizione della morfologia derivativa dell'italiano. Bologna: il Mulino.

Lo Duca, M. G. (2020). Italiano: la formazione delle parole. Roma: Carocci.

Masini, F. (2012). Parole sintagmatiche in italiano. Roma: Caissa Italia.

Micheli, M. S. (2020). La formazione delle parole. Italiano e altre lingue. Roma: Carocci.

Migliorini, B. (1963³). I prefissoidi. Il tipo aeromobile, radiodiffusione. In Id. (a cura di), Saggi sulla lingua del Novecento, (pp. 9-60). Firenze: Sansoni. 
Salvi, G., \& Vanelli, L. (2004). Nuova grammatica italiana. Bologna: il Mulino.

Scalise, S. (1995). La formazione delle parole. In L. Renzi, G. Salvi \& A. Cardinaletti (a cura di), Grande grammatica italiana di consultazione (vol. III, pp. 473-516). Bologna: il Mulino.

Scalise, S., \& Bisetto, A. (2008). La struttura delle parole. Bologna: il Mulino.

Sensini, M. (2018). Con metodo. Milano: Mondadori Education.

Serianni, L. (con la collaborazione di A. Castelvecchi) (2000²). Italiano. Grammatica, sintassi, dubbi, con un Glossario di G. Patota. Milano: Garzanti.

Serianni, L., Della Valle, V., \& Patota, G. (2017). Italiano Plurale. Grammatica e scrittura. Milano-Torino: Pearson Italia.

Sgroi, S. C. (2003). Per una ridefinizione di "confisso": composti confissati, derivati confissati, parasintetici confissati vs etimi ibridi e incongrui. Quaderni di Semantica, 24(I)/2003, 81-153.

Simone, R. (1997). Esistono verbi sintagmatici in italiano? In T. De Mauro \& V. Lo Cascio (a cura di), Lessico e grammatica. Teorie linguistiche e applicazioni lessicografiche (pp. 155-170). Roma: Bulzoni.

Thornton, A. M. (2004). Conversione. In M. Grossmann \& F. Rainer (a cura di), La formazione delle parole in italiano (pp. 501-533). Tübingen: Max Niemeyer. Zordan, R. (2019). A rigor di logica. Milano: Rizzoli Education. 